Portland State University

PDXScholar

2-1-1984

\title{
Coexistence and spinodal curves in directionally bonded liquids using the four-cluster approximation
}

\author{
Erik Bodegom \\ Portland State University \\ Paul H. Meijer
}

Follow this and additional works at: https://pdxscholar.library.pdx.edu/phy_fac

Part of the Physics Commons

Let us know how access to this document benefits you.

\section{Citation Details}

"Coexistence and spinodal curves in directionally bonded liquids using the four-cluster approximation," with P.H.E. Meijer, J. Chem. Phys. 80, 1617 (1984)

This Article is brought to you for free and open access. It has been accepted for inclusion in Physics Faculty Publications and Presentations by an authorized administrator of PDXScholar. Please contact us if we can make this document more accessible: pdxscholar@pdx.edu. 


\title{
Coexistence and spinodal curves in directionally bonded liquids using the four-cluster approximation
}

\author{
E. Bodegom and Paul H. E. Meijer \\ Physics Department, Catholic University of America, Washington, D. C. 20064 \\ and Center for Absolute Physical Quantities, National Bureau of Standards, Washington, D. C. 20234
}

(Received 19 April 1983; accepted 2 November 1983)

We derive the phase diagrams and spinodals of binary liquid systems with anisotropic interactions, such as hydrogen-bonded molecules. The work is based on the four-particle cluster variation method, using a different potential for different contact points. It is shown that the introduction of a cluster larger than previously used by Barker and Fock, leads to a considerable improvement in the shape of the phase diagram and avoids some of the difficulties encountered in their calculation. Phase diagrams are displayed for various choices of the parameters: the number of contact points, the interaction potential, and the order of the approximation.

\section{INTRODUCTION}

It is of interest to study the effects of the anisotropic microscopic interactions (e.g., via hydrogen bonding) on the limit of under- or supercooling, i.e., the spinodal line, since the amount of undercooling sustained is large in materials with anisotropic interactions. The anisotropic interactions also play an important role in the study of closed loop phase diagrams. Closed loop phase diagrams occur in various pairs of liquids, ${ }^{1}$ the mixture of water and nicotine being the classic example. At higher temperatures the two components can mix in any proportion, while at lower temperatures there is a certain range of concentrations of, for example, water and nicotine where the two liquids demix. At still lower temperatures, the components become again miscible in arbitrary proportions.

It is generally found that such phase diagrams are flatter at the bottom than at the top. The highest and lowest temperatures, where phase separation begins or ends, are called, respectively, the upper and lower critical solution temperatures (UCST and LCST). It is to be noted that in many systems the UCST and/or LCST cannot be observed because either a liquid-gas transition or a liquid-solid transition interferes, thus masking one of the critical solution temperatures.

Hirschfelder et $a l^{2}$ suggested that such a closed loop phase diagram was due to an interaction such as hydrogen bonding which interferes with the free rotation of the molecules. Barker and Fock ${ }^{3}$ were the first to show, using the quasichemical approximation, that such an interaction could indeed lead to closed loop phase diagrams. In the next section their work will be described and commented upon.

Another approach to the explanation of the closed loop phase diagrams has been undertaken by Andersen and Wheeler ${ }^{4,5}$; their work will be presented in Sec. III. More recent work is found in Refs. 6-9. The Sec. IV will deal with our higher order approximation. We will demonstrate how to calculate the spinodal line in the various approaches in $\mathrm{Sec}$. V. The remainder of this paper covers the results of the calculations performed and a discussion of these results.

\section{BARKER AND FOCK DESCRIPTION USING THE CVM}

Barker and Fock ${ }^{3,10}$ used a lattice model, wherein each molecule occupies a lattice site of a $z$-coordinated lattice. Throughout the remainder of this work, a simple cubic lattice is considered. Each molecule is presumed to have $z$ contact points, one (hereafter called the "special" contact point) is of one kind and the remaining ones $(z-1)$ of the other kind (the "normal" contact points). The nearest neighbor interaction energy between two molecules depends on which of the contact points of each molecule are involved in forming the "bond."

The two types of molecules are denoted by $A$ and $B$, each of which can be oriented in $z$ different directions (absolute directions in space). This can be conveniently described by an arrow, where the tip of the arrow indicates the special contact point. Thus for A molecules:

$$
f, \rightarrow,+, \text { etc. }
$$

and for B molecules:

$$
\uparrow, \rightarrow,+, \text { etc. }
$$

The statistical mechanics of such a system may lead to two kinds of possible states:

(a) The concentrations of the A molecules in the $z$ different ("absolute") directions are all equal, and the same holds true for the B molecules, or (b) the concentrations of the A molecules in the different orientations are not equal (the same holds true for the B molecules), i.e., there is a net orientation of the molecules (liquid crystals).

In this work, only the first case (the isotropic solution) will be considered. First, this model will be solved in the quasichemical approximation, as was done by Barker and Fock, but formulated in the cluster variation method $(\mathrm{CVM})^{11}$ and then by using the four point cluster approximation.

The CVM approach is as follows. On a given lattice, a basic figure or cluster is chosen. The basic cluster can be a point which leads to the mean field or Bragg-Williams approximation, or it can be the nearest neighbor pair which 
leads to the quasichemical or Bethe approximation or a larger cluster, such as a four point cluster consisting of a square on a cubic lattice. Each figure has a set of cluster variables associated with it. Each cluster variable indicates the probability of finding a certain configuration of $\mathrm{A}$ and $\mathrm{B}$ molecules on that particular figure. Denoting all the necessary cluster variables in a given approximation by $[\alpha]$, the free energy $(F)$ in the CVM can be expressed in terms of $[\alpha]$ :

$$
F[\alpha]=E[\alpha]-T S[\alpha],
$$

where $E, T$, and $S$ are, respectively, the internal energy, the absolute temperature, and the configurational entropy. The internal energy is given by the sum of the energies of all the clusters occurring in the system; for example, the sum over all the possible pair clusters of the product of the total number of pairs in the system and a pair probability and the energy associated with that particular pair. Under the assumption of pairwise additive and nearest neighbor forces, the expression for the energy is given exactly in the pair approximation. The entropy expression is obtained by taking the logarithm of the number of the possible ways one can distribute the $[\alpha]$ over the lattice sites. This number can not, in general, be found exactly. The CVM gives a prescription on how to approximate this entropy optimally for a given cluster size. After the expression for the free energy is obtained in terms of $[\alpha]$, the equilibrium free energy can be calculated by minimizing $F$ with respect to the $[\alpha]$.

To solve the Barker and Fock model in the quasichemical or pair approximation, the point and pair cluster variables are defined as follows. Let there be $n$ point variables $x_{i}^{\mathrm{A}}$ $(i=1, \ldots, n)$ for the A molecules. $n$ is the total number of possible orientations, which, for the moment, we will consider equal to $z$, the total number of neighbors. Here it is assumed that there is no orientational ordering (i.e., no liquid crystalline phases). Thus:

$$
x_{i}^{\mathrm{A}}=x_{\mathrm{A}} / n,
$$

where $x_{\mathrm{A}}$ is the concentration of the A molecules. A similar expression holds for the $\mathrm{B}$ molecules, with the normalization:

$$
x_{\mathrm{A}}+x_{\mathrm{B}}=1 .
$$

The pair variables $y_{i}(i=1, \ldots, 10)$ are presented in Table $\mathrm{I}$ together with the weight factors and the energies associated

TABLE I. The pair variables $\left(y_{i}\right)$ with the associated weight factors $\left(g_{i}\right)$ and energies $\left(\epsilon_{i}\right)$. The last column gives the expressions for their equilibrium values.

\begin{tabular}{lcccll}
\hline \hline & Bond & $g_{i}$ & $\epsilon_{i}$ \\
\hline$y_{1}$ & $\uparrow$ & $\uparrow$ & $(n-1)^{2}$ & 0 & $\left(x_{\mathrm{A}} x_{\mathrm{A}}\right)^{5 / 6} \mathrm{e}^{\beta \lambda}$ \\
$y_{2}$ & $\rightarrow$ & $\uparrow$ & $2(n-1)$ & 0 & $\left(x_{\mathrm{A}} x_{\mathrm{A}}\right)^{5 / 6} e^{\beta \lambda}$ \\
$y_{3}$ & $\rightarrow$ & $\leftarrow$ & 1 & 0 & $\left(x_{\mathrm{A}} x_{\mathrm{A}}\right)^{5 / 6} e^{\beta \lambda}$ \\
$y_{4}$ & $\uparrow$ & $\uparrow$ & $2(n-1)^{2}$ & $U_{1}$ & $\left(x_{\mathrm{A}} x_{\mathrm{B}}\right)^{5 / 6} e^{\beta \lambda-\beta \mathrm{U}_{1}}$ \\
$y_{5}$ & $\uparrow$ & + & $2(n-1)$ & $U_{2}$ & $\left(x_{\mathrm{A}} x_{\mathrm{B}}\right)^{5 / 6} e^{\beta \lambda-\beta U_{2}}$ \\
$y_{6}$ & $\rightarrow$ & $\uparrow$ & $2(n-1)$ & $U_{3}$ & $\left(x_{\mathrm{A}} x_{\mathrm{B}}\right)^{5 / 6} e^{\beta \lambda-\beta U_{3}}$ \\
$y_{7}$ & $\rightarrow$ & + & 2 & $U_{4}$ & $\left(x_{\mathrm{A}} x_{\mathrm{B}}\right)^{5 / 6} e^{\beta \lambda-\beta U_{4}}$ \\
$y_{8}$ & $\uparrow$ & $\uparrow$ & $(n-1)^{2}$ & 0 & $\left(x_{\mathrm{B}} x_{\mathrm{B}}\right)^{5 / 6} e^{\beta \lambda}$ \\
$y_{9}$ & $\rightarrow$ & $\uparrow$ & $2(n-1)$ & 0 & $\left(x_{\mathrm{B}} x_{\mathrm{B}}\right)^{5 / 6} e^{\beta \lambda}$ \\
$y_{10}$ & $\rightarrow$ & $\rightarrow$ & 1 & 0 & $\left(x_{\mathrm{B}} x_{\mathrm{B}}\right)^{5 / 6} e^{\beta \lambda}$ \\
\hline \hline
\end{tabular}

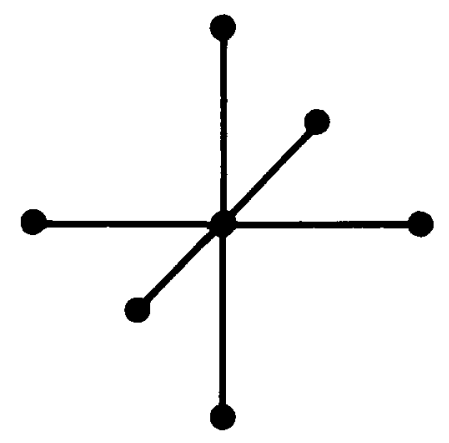

FIG. 1. The smallest basic cluster size needed to automatically incorporate the constraints (2).

with them. It is assumed that the energy is zero between like molecules independent of orientation as in Barker and Fock. The pair variables obey the following relations:

$$
\sum_{i=1}^{10} g_{i} y_{i}=1
$$

and

$$
\begin{aligned}
x_{\mathrm{A}}= & (n-1)^{2} y_{1}+2(n-1) y_{2}+y_{3} \\
& +(n-1)^{2} y_{4}+(n-1) y_{5}+(n-1) y_{6}+y_{7}, \\
x_{\mathrm{B}}= & (n-1)^{2} y_{8}+2(n-1) y_{9}+y_{10} \\
& +(n-1)^{2} y_{4}+(n-1) y_{5}+(n-1) y_{6}+y_{7} .
\end{aligned}
$$

In addition, two more relations are needed to account for the fact that for each type of molecule the ratio of the number of special contact points to all contact points is $1 / n$, that is for the A molecules:

$$
\frac{1}{n}=\frac{(n-1) y_{2}+y_{3}+(n-1) y_{6}+y_{7}}{x_{\mathrm{A}}} \equiv R_{\mathrm{A}},
$$

and for the B molecules:

$$
\frac{1}{n}=\frac{(n-1) y_{9}+y_{10}+(n-1) y_{5}+y_{7}}{x_{\mathrm{B}}} \equiv R_{\mathrm{B}} \text {. }
$$

These additional relations are needed owing to the fact that the cluster size considered here is not large enough. The smallest cluster size large enough to automatically fullfil the conditions (2) is the basic cluster shown in Fig. 1. The problem with this cluster is that it leads to a number of the independent basic cluster variables that is almost prohibitively large. So, at first sight, it seems quite reasonable to assume that the constraints have to be incorporated in the final solution, as was done by Barker and Fock.

Here however we will follow a different route. The constraints will be ignored, but by going to a larger cluster size the influence of this neglect will be diminished. This is a compromise. The difficulty would have been totally taken care of if we had used the seven-point cluster of Fig. 1.

In general $\Phi(=\beta F$, where $F$ is the free energy and $\beta=1 / k T)$ is given by ${ }^{11}$

$$
\begin{aligned}
\Phi= & 3 \beta \sum_{i=1}^{10} \epsilon_{i} g_{i} y_{i}-5\left[x_{\mathrm{A}}\left(\ln x_{\mathrm{A}}-1\right)+x_{\mathrm{B}}\left(\ln x_{\mathrm{B}}-1\right)\right] \\
& +3 \sum_{i=1}^{10} g_{i} y_{i}\left(\ln y_{i}-1\right)+3 \beta \lambda\left(1-\sum_{i=1}^{10} g_{i} y_{i}\right),
\end{aligned}
$$

where $\lambda$ is the Lagrange multiplier for the normalization of the probabilities. Additional Lagrange multipliers are 
needed if one wants to include the constraints given by Eqs. (2). This we will not do. Following the usual procedure of minimizing $\Phi$ with respect to the variables $y_{i}$ leads to equations of the form (see also Table I):

$$
y=(x x)^{5 / 6} e^{\beta \lambda-\beta \epsilon} .
$$

Note that the pair probabilities can be classified in three groups. One group consists of $y_{1}, y_{2}$, and $y_{3}$ (we refer to these as $\left.y_{1}^{\prime}\right)$ and the second group consists of $y_{8}, y_{9}$, and $y_{10}\left(y_{3}^{\prime}\right)$. The third group $\left(y_{4}, y_{5}, y_{6}\right.$, and $\left.y_{7}\right)$ can be replaced by $y_{2}^{\prime}$ by using

$$
n^{2} y_{2}^{\prime}=(n-1)^{2} y_{4}+(n-1) y_{5}+(n-1) y_{6}+y_{7} .
$$

Putting

$$
y_{2}^{\prime}=e^{\beta \lambda-K}\left(x_{\mathrm{A}} x_{\mathbf{B}}\right)^{5 / 6},
$$

where $K$ represents the effective interaction energy between $\mathrm{A}$ and $\mathrm{B}$ molecules times $\beta$. It follows that $K$ has to satisfy:

$$
\begin{aligned}
n^{2} e^{-K}= & (n-1)^{2} e^{-\beta U_{1}} \\
& +(n-1) e^{-\beta U_{2}}+(n-1) e^{-\beta U_{3}}+e^{-\beta U_{4}} .
\end{aligned}
$$

The primed system is entirely analogous to a magnetic spin-1/2 system with the effective coupling $K / 2$ replacing $\beta \epsilon .{ }^{11}$ The UCST and LCST are determined by the following condition ${ }^{12}$ :

$$
K_{\text {critical }}=\ln \frac{z}{z-2} \text {. }
$$

Phase separation occurs when $K>K_{c}$ and the phase diagrams resulting from specific choices of the energies $U_{i}$ can easily be calculated (see Appendix A). The results will be presented in the last section after several other approaches have been discussed. The results will be compared with the work of Andersen and Wheeler. ${ }^{4,5}$

\section{ANDERSEN AND WHEELER MODELS}

The closed loop phase diagrams calculated with the Barker and Fock method have two serious drawbacks: one is that they are too "narrow" compared to coexistence curves observed on real physical systems; and the other is that the predicted interaction energies for the hydrogen bonding are far too low compared with known experimental hydrogen bond strengths. ${ }^{14,15}$

Wheeler ${ }^{13}$ proposed to use a decorated lattice model which can be mapped onto the Ising model. This model showed a considerable widening of the phase diagrams and a better hydrogen bond interaction energy. Although this results in an improvement over the method of Barker and Fock, it failed on the same account as the Barker and Fock model in comparison with real physical systems. As was pointed out by Andersen and Wheeler, ${ }^{4}$ there is another parameter which, so far, has been ignored, i.e., the directionality of the special contact point. Both Wheeler and Barker and Fock assumed implicitly that the directionality is determined by the underlying lattice. In other words, the solid angle subtended by the special contact point is equal to $4 \pi / 6$ (for $z=6$ ). This is by far too large compared to what is known from measurements, ${ }^{14.15}$ which show that the polar angle is in the order of $15^{\circ}$.
To study the influence of the directionality of the special contact point, Andersen and Wheeler ${ }^{4,5}$ extended the decorated lattice model of Wheeler. ${ }^{9}$ In this model there are two kinds of sites: one kind, the primary sites or cells, is located on the principal lattice (again a cubic lattice was used); and the other kind, the secondary sites, are introduced between each of the two nearest neighbor primary cells. As in the Barker and Fock model, either an A or B molecule occupies every site. It is now assumed that the only interaction is between nearest neighbor primary and secondary cells. Each molecule is again represented by an arrow which now, in contrast to the Barker and Fock model, can point in $n$ directions where $n$ is an assignable number. The interaction energies are chosen as follows. Between molecules of the same species, the energy is zero; and between unlike molecules, the interaction depends on whether the arrow, on the secondary cell, points towards or away from the primary cell. It is assumed that pointing towards the primary cell occupant constitutes a hydrogen bonding $\left(U_{2}\right)$, where $U_{2}$ is negative (i.e., an attractive interaction), and pointing away gives the repulsive energy $U_{1}$.

It should be emphasized that the energy in the Andersen and Wheeler model does not depend at all on the orientation of the molecule on the primary site. This limitation had to be imposed, otherwise the model cannot be solved by mapping it onto the Ising model. The general outline for solving a decorated lattice is given in Mulholland and Rehr. ${ }^{16}$

The calculation of closed loop coexistence curves by this method shows that, in order to get a good fit with the experimental curves, $n$ should be equal to 5000 . This puts the directionality at a much too low value. Andersen et al. reason that this is not surprising since the directionality of the molecules on the primary sites is ignored. They estimate that the correct solid angle subtended by the special contact point is calculated from an effective $n$, which they take to be $\sqrt{n}$. Reasonable agreement is now obtained for the solid angle.

Although the decorated system has provided good agreement with the experimental curves, the artificial feature that molecules on primary sites have no directionality associated with them is a severe drawback. As is often argued $^{17,18}$ it is more likely that a network develops; that is to say, there exists a certain correlation between these orientations.

The treatment of the Barker and Fock model in Sec. II can be extended in order to incorporate a higher directional bonding. In order to do this, one would have to differentiate between two kinds of $A$ and $B$ molecules, i.e., those molecules that form a hydrogen bond with their neighbors and those that do not, as was done by Andersen and Wheeler. As mentioned before we will ignore the constraints (2) and as a consequence some of the need for the differentiation falls away. In addition, our primary interest is in the determination of the influence of directional bonding on the spinodal line and not the complete solution of the model. Thus we ignored the distinction referred to above.

Now it becomes easy to extend the Barker and Fock model to include the stronger directional bonding. $n$ is no 
longer equal to $z$, as it was in the previous section, but can vary. From Eq. (6), the consequences are clear. Renaming the energies in Eq. (6) to conform with the notation of Andersen and Wheeler (i.e., the repulsive energy $U_{1} \equiv U_{1}$ and the attractive energy $U_{2} \equiv U_{2}=U_{3}=U_{4}$ ), it is seen that an increase in $n$ leads to a relative decrease of the importance of the term with the attractive energy. Thus, comparing two cases with the same energies but with different values for $n$, one finds that the larger the value for $n$, the lower the LCST. Or, in other words, to achieve the same ratio of UCST and LCST, a larger attractive energy is needed in the case with the larger $n$. Thus, a more realistic value is found for the hydrogen bond energy. However, as before, the coexistence curves are still too narrow, which might be argued to be due to the effect that the order of the approximation is too low in the Barker and Fock model. In the next section, the next higher order approximation in the CVM (the four cluster or Kramers-Wannier ${ }^{19}$ approximation) will be treated.

\section{THE FOUR CLUSTER APPROXIMATION}

Using the same arguments as in the previous sections, it is assumed that all the point probabilities in the $n$ directions are equal. Consequently, the number of the possible four cluster variables (see Fig. 2), namely $n^{4}$, is reduced to, at most, $6^{4}$ different (or independent) variables. An added reduction can be achieved using symmetries. In the natural iteration $^{20}$ method (NI), as in many other methods, the smaller the number of the independent basic cluster variables, the easier it is to solve the system (see Appendix B).

Let the indices $i, j, k$, and $l$ denote the absolute orientations in space of either the $\mathrm{A}(i, \ldots=1, \ldots, n)$ or $\mathrm{B}$ $(i, \ldots=n+1, \ldots, 2 n)$ and let $x_{i}, y_{i j}$, and $z_{i j k l}$ denote, respectively, the point, pair, and four cluster variables. In the isotropic solution, all the $x_{i}$ for $i \leqslant n$ are equal to $x_{\mathrm{A}} / n$. The list of the different pair variables is given in Table $I$. The four point variables are not listed. They were actually generated in the program. $\Phi(=\beta F)$ is then given by

$$
\begin{aligned}
\Phi= & 3 \beta \sum_{i j=1}^{2 n} \epsilon_{i j} y_{i j}+7 \sum_{i=1}^{2 n} x_{i}\left(\ln x_{i}-1\right) \\
& -9 \sum_{i j=1}^{2 n} y_{i j}\left(\ln y_{i j}-1\right)+3 \sum_{i, j, k=1}^{2 n} z_{i j k l}\left(\ln z_{i j k l}-1\right) \\
& +3 \beta \lambda\left(1-\sum_{i j, k, l=1}^{2 n} z_{i j k l}\right) .
\end{aligned}
$$

Minimizing with respect to $z_{i j k l}$ and remembering that

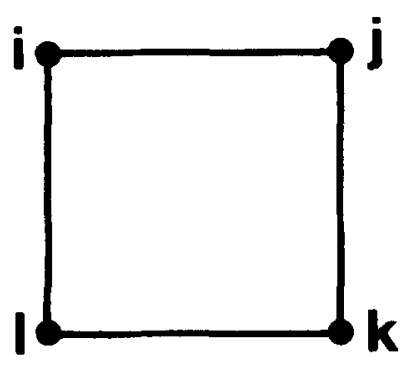

$$
x_{i}=\sum_{j} y_{i j}=\sum_{j, k, l} z_{i j k l},
$$

and

$$
y_{i j}=\sum_{k l} z_{i j k l},
$$

leads to

$$
\begin{aligned}
z_{i j k l}= & \left(x_{i} x_{j} x_{k} x_{l}\right)^{-7 / 12}\left(y_{i j} y_{j k} y_{k l} y_{l i}\right)^{3 / 4} \\
& \times\left[-\beta\left(\lambda+\frac{\epsilon_{i j}+\epsilon_{j k}+\epsilon_{k l}+\epsilon_{l i}}{4}\right)\right],
\end{aligned}
$$

where, for convenience, the energies in Table I have been renamed. Equation (10) can be written as

$$
z_{i j k l}=A_{i j} A_{j k} A_{k l} A_{l i} e^{-\beta \lambda}
$$

with

$$
A_{i j}=\left(x_{i} x_{j}\right)^{-7 / 24} y_{i j}^{3 / 4} e^{-\beta \epsilon_{i j} / 4} .
$$

Inspection of the set of Eqs. (11) reveals that the $(2 n)^{4} z_{i j k l}$ can be reduced to only 124 different four cluster variables with weight factors.

Having established how the Barker and Fock model (with the extension of the Anderson and Wheeler model) is to be solved in two different approximations for the equilibrium cases, it is now possible to calculate the spinodal as outlined in the following section.

\section{THE CALCULATION OF THE SPINODAL}

The spinodal ${ }^{21}$ is determined by

$$
\frac{d^{2} F}{d \xi^{2}}=0
$$

where $\xi=x_{\mathrm{A}}-x_{\mathrm{B}}$. This is easily calculated in a mean field theory where $\zeta$ is the only variable. For the pair and the four cluster approximations, Eq. (12) becomes a complicated expression in the pair and the four cluster variables, respectively. It will be shown that the condition (12) is the same as the condition that the determinant, formed by the matrix of the second derivatives of the free energy with respect to the independent variables, vanishes.

We will demonstrate the calculation using the pair approximation, particularly since the results published in the literature are incorrect. Let us consider a magnetic or a simple phase separating system in the pair approximation. The grand potential $\Phi=\beta\left[F+H\left(x_{1}-x_{2}\right)\right]$ is given by

$$
\begin{aligned}
\Phi= & \frac{z}{2} \beta \epsilon\left(2 y_{2}-y_{1}-y_{3}\right)-(z-1) \sum_{i=1}^{2} x_{i}\left(\ln x_{i}-1\right) \\
& +\frac{z}{2} \sum_{i=1}^{3} g_{i} y_{i}\left(\ln y_{i}-1\right)+\beta H\left(x_{1}-x_{2}\right),
\end{aligned}
$$

where $x_{1}, x_{2}, y_{1}, y_{2}$, and $y_{3}$ are, respectively, the probabilities for spin up, spin down, two nearest neighbor spins up, spin up, and spin down, and the two n.n. spins down and $g_{i}$ equals 2 if $i=2$ and $g_{i}$ equals 1 otherwise. $z$ is the coordination number and $H$ is the magnetic field or the chemical potential. The interaction energy is chosen such that one does not have to determine the slope of the common tangent. $^{22}$ 
The following relations hold between the probabilities:

$$
\begin{aligned}
& x_{1}+x_{2}=1, \\
& x_{1}=y_{1}+y_{2}, \\
& x_{2}=y_{2}+y_{3} .
\end{aligned}
$$

Taking $\xi\left(=x_{1}-x_{2}\right)$ and $y_{2}$ as independent variables, the remaining probabilities can be expressed as

$$
\begin{aligned}
& x_{1}=\frac{1}{2}(1+\xi), \\
& x_{2}=\frac{1}{2}(1-\xi), \\
& y_{1}=\frac{1}{2}(1+\xi)-y_{2}, \\
& y_{3}=\frac{1}{2}(1-\xi)-y_{2} .
\end{aligned}
$$

The grand potential is a minimum with respect to a variation in the variables, consequently

$$
\begin{aligned}
& \frac{\partial \Phi}{\partial \xi}=0=-\frac{z-1}{2} \ln \frac{x_{1}}{x_{2}}+\frac{z}{4} \ln \frac{y_{1}}{y_{3}}+\beta H \equiv S_{1}, \\
& \frac{\partial \Phi}{\partial y_{2}}=0=2 z \beta \epsilon+\frac{z}{2} \ln \frac{y_{2}^{2}}{y_{1} y_{3}} \equiv S_{2} .
\end{aligned}
$$

The first equation determines the magnetic field. A variational principle ${ }^{23}$ says that

$$
d S_{2}=0=\frac{\partial S_{2}}{\partial \xi} d \xi+\frac{\partial S_{2}}{\partial y_{2}} d y_{2}
$$

or

$$
\frac{\partial S_{2}}{\partial y_{2}} \frac{d y_{2}}{d \xi}+\frac{\partial S_{2}}{\partial \xi}=0
$$

A short calculation shows that (indicating the partial derivatives by indices):

$$
\frac{d^{2} \Phi}{d \xi^{2}}=\left(\Phi_{\xi \xi} \Phi_{y_{2} y_{2}}-\Phi_{y_{2} \xi}^{2}\right) / \Phi_{y_{2} y_{2}}=0
$$

where $\Phi_{y_{2} y_{2}}$ does not equal zero [see Eq. (17)]. This is of course the matrix of the free energy with respect to the independent variables.

For the magnetic case, the following expressions result from Eq. (18) with Eqs. (15) and (16):

$$
\begin{aligned}
& y_{2}=\frac{z-2}{z-1} x_{1} x_{2}, \\
& e^{4 \beta \epsilon}=\frac{y_{1} y_{3}}{y_{2}^{2}} .
\end{aligned}
$$

This can be rewritten as follows:

$$
\begin{aligned}
\frac{T}{T_{c}}= & 2 \ln \left(\frac{z}{z-2}\right) \\
& \times\left[\ln \frac{(z-2)\left(1-\xi^{2}\right)+4(z-1) /(z-2)}{(z-2)\left(1-\xi^{2}\right)}\right]^{-1} .
\end{aligned}
$$

This result is to be compared with an expression recently derived by van der Haegen et al. ${ }^{24}$ :

$\frac{T}{T_{c}}=\ln \left(\frac{z}{z-2}\right)\left\{\ln \frac{A+\left[A^{2}+4 z(z-2)\right]^{1 / 2}}{2(x-2)}\right\}^{-1}$

with

$$
A=\left(\frac{1+\xi}{1-\xi}\right)^{(z-1) / z}+\left(\frac{1-\xi}{1+\xi}\right)^{(z-1) / z}
$$

since they incorrectly used the equilibrium free energy (i.e., $H=0$ ).

By the same reasoning, for a system which has more than two independent variables, one can show that the condition for the spinodal line is the vanishing of the determinant of the derivatives (see also Meijer et al. ${ }^{25}$ ).

Since in the four cluster approximation there are $124-1$ (from the normalization condition) $=123$ independent variables, it is often more convenient from a computational point of view not to calculate the determinant but to determine by trial and error where the magnetic field reaches an extreme inside the coexistence curve, subject to the set of equations (16).

In the next section the results of the calculations will be discussed.

\section{RESULTS AND DISCUSSION}

In Fig. 3 the upper and lower critical temperatures are plotted for the pair and the four cluster approximations as a function of $U_{2}$. Here it is assumed that $U_{3}=U_{4}=U_{2}$, where $U_{2}$ is the hydrogen bonding interaction. $U_{1}$ is arbi-

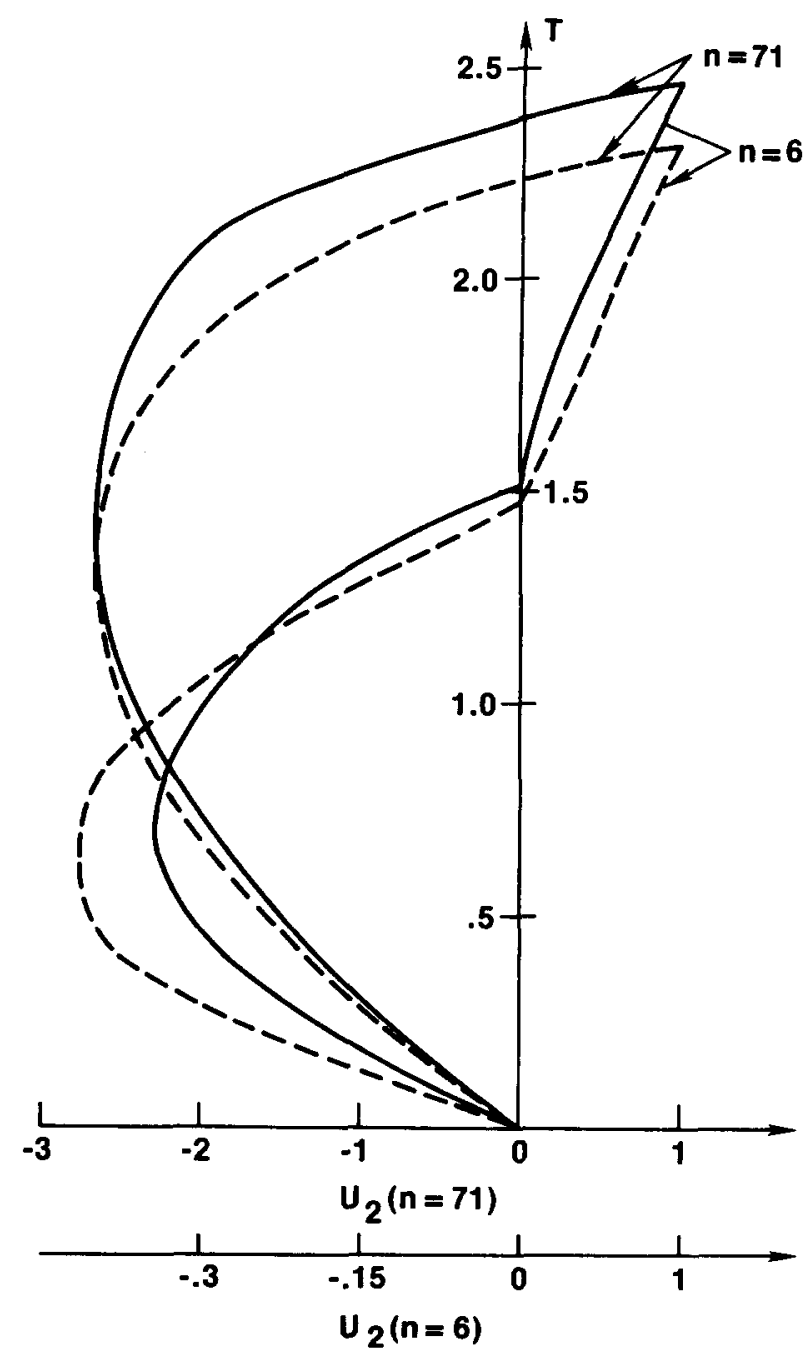

FIG. 3. Upper and lower critical solution temperatures plotted as function of the energy $U_{2}$ for two different values of $n$. $U_{1}$ has been taken equal to one. The solid lines refer to the pair approximation and the dotted lines refer to the four cluster approximation. 


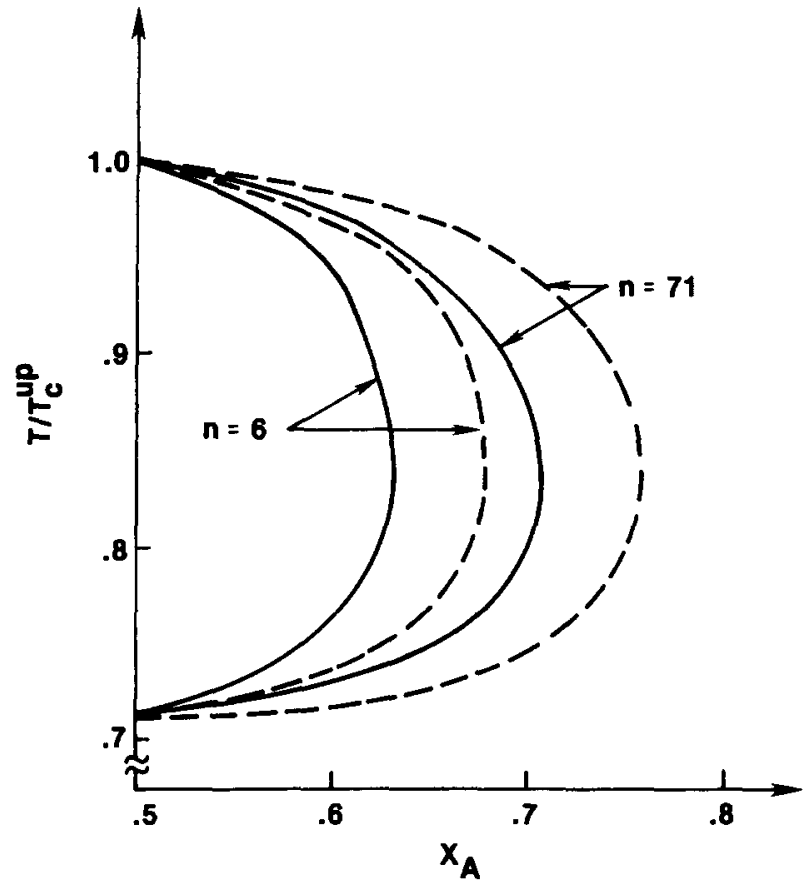

FIG. 4. Coexistence curves for pair ( = solid lines) and four cluster ( = dotted lines) approximations for two different values of $n$. Since the coexistence curves are symmetric around $x_{\mathrm{A}}=\mathbf{0 . 5}$, only half of the phase diagrams are drawn. The energies $U_{2}\left(U_{1}=1\right)$ and the UCST are as follows: Pair, $n=6$ : $U_{2}=-0.339, T_{c}^{\text {up }}=0.815$. Pair, $n=71: U_{2}=-2.60, T_{c}^{\text {up }}=1.67$. Four cluster, $n=6: \quad U_{2}=-0.414, \quad T_{c}^{\text {up }}=0.75$. Four cluster, $n=71$ : $U_{2}=-2.58, T_{c}^{\mathrm{up}}=1.53$.

trarily chosen to be equal to one, so it determines the temperature scale. The additional parameter labeling the curves indicates how strong the anisotropy is, i.e., the value of $\boldsymbol{n}$. It is obvious that an increase in $\boldsymbol{n}$ gives an increase in the ratio of upper to lower critical solution temperatures as was noted earlier by Andersen and Wheeler. The influence of the approximation, pair vs square, on the curves in Fig. 3 is seen to be rather small.

However, a large influence of the approximation can be noted if the actual coexistence curves are plotted. To make a meaningful comparison possible, the coexistence curves were calculated such that the ratio of upper to lower critical solution temperatures is the same. The results are shown in Fig. 4. As was mentioned before, a higher order approximation gives a considerable widening of the coexistence curves over the quasichemical approximation.

Moreover, as anticipated, the conditions described in Eq. (2) were better fulfilled with the higher order approximation (see Fig. 6). It is worth noting that it is expected that

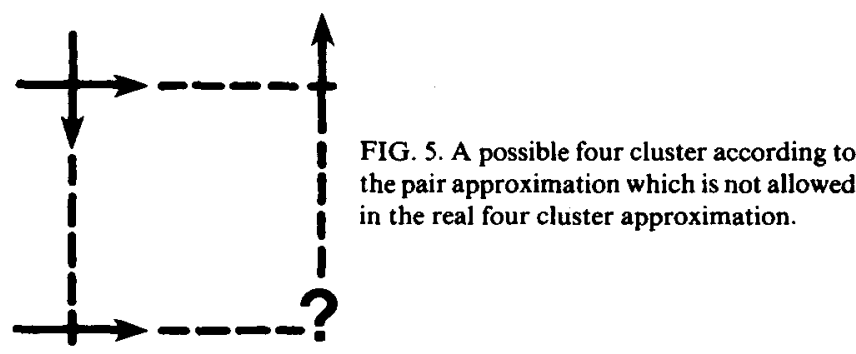

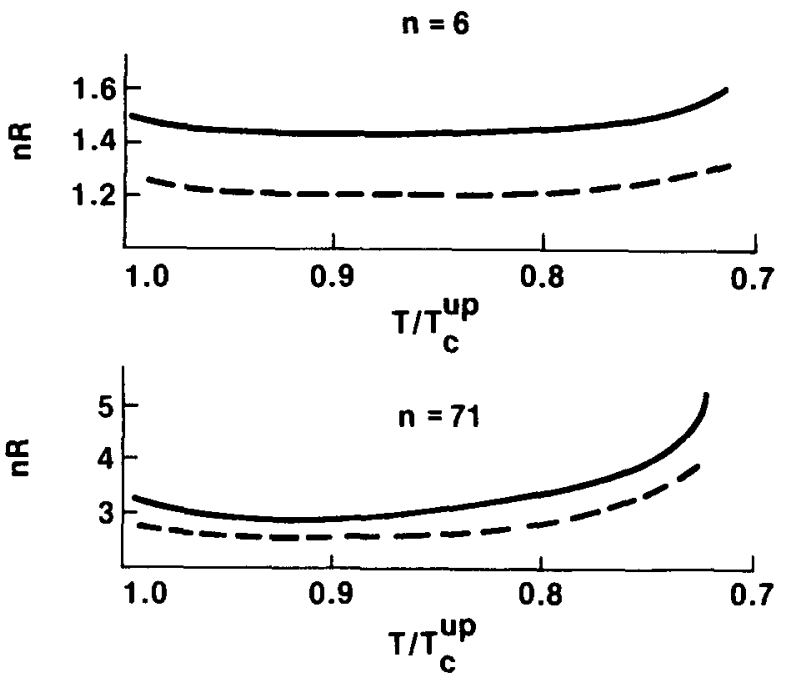

FIG. 6. The ratio of special to all contact points for the $A$ molecules $\left(R_{A}\right)$ as a function of reduced temperature for the coexistence curves shown in Fig. 4. Solid lines are for the pair approximation and the dotted lines indicate the four cluster approximation.

this ratio will be more nearly $1 / 6$ in the case $n=6$ in the four cluster approximation than in the pair approximation. This is due to the fact that in the four cluster approximation certain figures are eliminated because they are inconsistent with the model. In other words, using the pair approximation a certain number of inconsistent four clusters are allowed. For a specific example see Fig. 5. This four cluster is possible in the pair approximation but impossible in the four cluster approximation. That is, the four cluster approximation is closer to the minimum cluster size (see Fig. 1) needed to adequately describe the model. In the model with $n$ larger than 6 , this ratio remains $1 / n$. But, it has to be

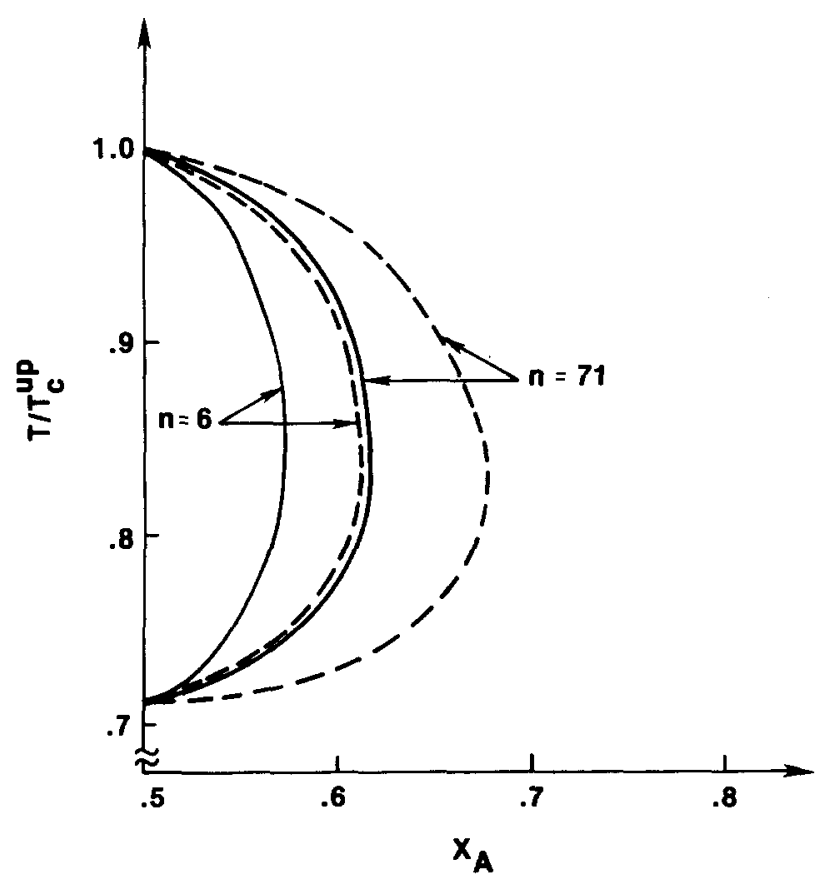

FIG. 7. The spinodal lines of the coexistence curves given in Fig. 4 (solid and dotted lines are for, respectively, the pair and four cluster approximations). 
remembered that, here, an additional approximation is made (see Sec. III). Thus, the values of the ratios for different $n$ are not comparable. The results show that these arguments are confirmed (see Fig. 6). The ratios, in the case $n$ larger than 6, are shown to be much less constant than for $n$ equal to 6 . This is not surprising, since more approximations are made in the first case.

In Fig. 7 the spinodal lines are presented for the same coexistence curves as in Fig. 4. It is noticed that the spinodals are wider if either the order of the approximations or the anisotropy (as measured by $n$ ) increases. In Sec. II it was pointed out that in the pair approximation, the coexistence curve could be mapped onto the Ising model with a temperature dependent interaction energy. Since, in the pair approximation for a nonanisotropic system, one specific temperature corresponds to one specific concentration at the coexistence curve and also corresponds to one specific concentration at the spinodal, the appropriate way of comparing the different spinodal lines is as follows. For a given concentration at the coexistence curve, one calculates both the spinodals for the closed loop phase diagram and the magnetic system. This means, of course, that besides the fact that the temperatures (i.e., their numerical values) are different, also the reduced temperatures (i.e., $T / T_{\text {uc }}$, where $T_{\mathrm{uc}}$ is the temperature at the upper critical solution point) will, in general, be different. It is found that, for each approximation considered here, the spinodals are approximately the same (i.e., the difference is less than $0.02 \%$ ). This is unexpected since, if one considers the pair approximation, the determinant of the matrix of the derivatives (which determines the spinodal curve) is entirely different in both cases (for anisotropic molecules one has a $(3 \times 3)$ matrix and for isotropic molecules or a magnetic system one has a $(2 \times 2)$ matrix). There is, however, a small dependence on the approximation used. However, as is believed, ${ }^{26}$ the van der Waals loop is a consequence of the approximation. The van der Waals loop is supposed to disappear in the correct solution. But this corresponds, in the CVM, to an infinitely large cluster which is computationally impossible. The question is whether the spinodals calculated from an equilibrium theory, such as the CVM, have any meaning beyond appearing to be a rather fortuitous coincidence. It is well known, that nucleation is a time dependent process ${ }^{27}$; thus, it maybe necessary to generalize the problem into the time domain.

\section{SUMMARY}

It is pointed out that the Barker and Fock model should be solved in a much higher approximation to better account for the anisotropy of the molecules. It is also shown that, using a higher order approximation than previously used, a considerable widening of the closed loop phase diagram is obtained which is more in accordance with real physical systems. Also, in the same higher order approximation, the ratio of the special to all contact points is shown to be closer to the correct value.

Moreover, it is noted that in real systems the anisotropy of the molecules gives rise to the formation of liquid crystals (and/or complicated networks) which, in any of the models discussed so far, cannot be accounted for. However, in the CVM, the formation of liquid crystals can be included. The results can be compared with recent experiments ${ }^{28}$ in which, in addition to a closed loop phase diagram, liquid crystalline phases were also found.

After having corrected a recently published expression for the spinodal line, the spinodals are calculated in both the pair and four cluster approximations. It is found that, within an approximation, the influence of the anisotropy of the molecules is small to neglible.

\section{APPENDIX A: SOLUTION OF THE EQUATIONS IN THE PAIR APPROXIMATION}

The equilibrium concentration is calculated by using the following substitution ${ }^{12}$ :

$$
x_{\mathrm{A}} / x_{\mathrm{B}}=e^{6 \theta} \text {. }
$$

This leads to

$$
\xi=x_{\mathrm{A}}-x_{\mathrm{B}}=\tanh 3 \theta .
$$

The minimization of the free energy, with respect to the independent variables Eq. (3), leads to

$$
\begin{aligned}
& \frac{\partial \Phi}{\partial \xi}=0=-\frac{5}{2} \ln \frac{x_{\mathrm{A}}}{x_{\mathrm{B}}}+\frac{3}{2} \ln \frac{y_{1}^{\prime}}{y_{3}^{\prime}}, \\
& \frac{\partial \Phi}{\partial y_{2}^{\prime}}=0=6 K+3 \ln \frac{y_{2}^{\prime 2}}{y_{1}^{\prime} y_{3}^{\prime}} .
\end{aligned}
$$

Rewriting this in terms of the variable $\theta$ leads to

$$
e^{K}=\frac{\sinh 3 \theta}{\sinh 2 \theta}
$$

and

$$
y_{2}^{\prime}=\frac{1}{2} \frac{\sinh 2 \theta}{\sinh 5 \theta \cosh 3 \theta} .
$$

Equation (A4a) can be solved in several ways. ${ }^{23}$

\section{APPENDIX B: SOLVING THE FOUR CLUSTER EQUATIONS}

The weight factors were determined by systematic counting on the computer. ${ }^{29}$ The normal procedure for solving the equations resulting from the CVM is by means of the NI. ${ }^{20}$ Acceleration of the convergence is made possible by means of the following procedure. When the system is almost converged, the variables change logarithmically with each iteration, this knowledge can be used to extrapolate to the solution. ${ }^{22}$

The natural iteration technique of Kikuchi was later amended, by the same author, with the so-called minor iterations. ${ }^{30}$ This addendum was introduced to satisfy additional constraints which occurred in more complicated problems. These constraints give rise to additional Lagrange multipliers. The natural or major iterations are completed as outlined above. The minor iterations are different. After each calculation of the cluster variables, in their canonical form, one solves for the additional Lagrange multipliers (i.e., the minor iteration) and then proceeds with a major iteration followed, again, by minor iter- 
ations. These Lagrange multipliers will be seen to occur in the form:

$$
z_{i}=z_{i}^{(0)} e^{\alpha_{j}},
$$

where the $\alpha_{j}$ are the Lagrange multipliers and $z_{i}^{(0)}$ is the canonical part (i.e., the product of different subclusters) of the cluster variable $z_{i}$.

A new iteration scheme is proposed, where, both the minor and major iterations are treated on the same footing, i.e., minor and major iterations are done at the same time. For this to be achieved, the following transformation is proposed. First, it is to be noted that, according to the Barker and Fock model, for example, in the four cluster approximation there are only nine independent variables. This can be seen from the set of equations (11). The four point probabilities $\left(z_{j}\right)$ and the point probabilities $(x)$ are completely determined once all the pair probabilities are specified. Since there are ten pair probabilities with one normalization condition, there are only nine independent variables. The pair probabilities are now rewritten in terms of nine new variables $\left(r_{i}\right)$ as listed below:

$$
\begin{aligned}
& y_{1}=\exp \left(r_{1}+r_{2}+r_{3}\right), \\
& y_{2}=\exp \left(r_{1}+r_{2}-r_{3}+r_{4}\right), \\
& y_{3}=\exp \left(r_{1}+r_{2}-r_{3}-r_{4}\right), \\
& y_{4}=\exp \left(-r_{1}+r_{7}\right), \\
& y_{5}=\exp \left(-r_{1}-r_{7}+r_{8}\right), \\
& y_{6}=\exp \left(-r_{1}-r_{7}-r_{8}+r_{9}\right), \\
& y_{7}=\exp \left(-r_{1}-r_{7}-r_{8}-r_{9}\right), \\
& y_{8}=\exp \left(+r_{1}-r_{2}+r_{5}\right), \\
& y_{9}=\exp \left(r_{1}-r_{2}-r_{5}+r_{6}\right), \\
& y_{10}=\exp \left(r_{1}-r_{2}-r_{5}-r_{6}\right) .
\end{aligned}
$$

The point probabilities, expressed as a sum over pair probabilities, are seen not to depend strongly on any of the new variables $r_{i}$. The four cluster variables $(z)$ can be calculated using Eqs. (10) and, as in the NI, the new pair probabilities are formed using the new set of $z$. From these new pair probabilities one can calculate the new $r_{i}$ by means of Eqs. (B2). Schematically, one can write: $r_{i}=f(y)$, where $y$ indicates the approximate pair probabilities. So far the procedure is entirely analogous to the NI, but now certain improvements can be made. The variable that governs phase separation is $r_{2}$ (i.e., if $r_{2}$ is zero then $x_{\mathrm{A}}=x_{\mathrm{B}}$ ). This is the variable which, especially close to the critical point, changes slowly to the correct value. Thus, an improvement which can be imple- mented rather easily is changing $r_{2}$ faster than the change which would occur if one would adhere to the NI. Similarly, all the other variables could be changed faster than in the NI. This is a standard problem (see Henrici ${ }^{31}$ ). A comparison between the natural iteration with acceleration ${ }^{22}$ and the scheme proposed here for the four cluster approximation reveals that the latter is somewhat faster (a 20\% improvement).

\section{ACKNOWLEDGMENTS}

We would like to thank the Office of Naval Research for financial support. The calculations were made possible with the help of the CUA Computer Center. We would like to thank Dr. van der Haegen and Dr. Lekkerkerker for pointing out an arithmetical error in Eq. (20) in the original version of this paper.

'A. W. Francis, Critical Solution Temperatures, Adv. Chem. Ser. No. 31 (American Chemical Society, Washington, D.C. 1961).

${ }^{2}$ J. Hirschfelder, D. Stevenson, and H. Eyring, J. Chem. Phys. 5, 896 (1937).

${ }^{3}$ J. A. Barker and W. Fock, Discuss. Faraday Soc. 15, 188 (1953).

${ }^{4}$ G. R. Andersen and J. C. Wheeler, J. Chem. Phys. 69, 2082 (1978).

${ }^{5}$ G. R. Andersen and J. C. Wheeler, J. Chem. Phys. 69, 3403 (1978)

${ }^{6} \mathrm{~J}$. C. Wheeler and G. R. Andersen, J. Chem. Phys. 73, 5778 (1980).

${ }^{7}$ J. S. Walker and C. A. Vause, Phys. Lett. A 79, 421 (1980).

${ }^{8}$ C. A. Vause and J. S. Walker, Phys. Lett. A 90, 419 (1982).

${ }^{9}$ R. E. Goldstein and J. S. Walker, J. Chem. Phys. 78, 1492 (1983).

${ }^{10}$ J. A. Barker, J. Chem. Phys. 20, 1526 (1952).

${ }^{1}$ R. Kikuchi, J. Phys. (Paris) 38, C7-307 (1977).

${ }^{12}$ R. Kikuchi, Hughes Research Labs, unpublished, 1979.

${ }^{13}$ J. C. Wheeler, J. Chem. Phys. 62, 433 (1975).

${ }^{14}$ M. Joesten and L. Schaad, Hydrogen Bonding (Dekker, New York, 1974).

${ }^{15}$ S. N. Vinogradov and R. H. Linnell, Hydrogen Bonding (Van Nostrand Reinhold, New York, 1971).

${ }^{16} \mathrm{G}$. W. Mulholland and J. J. Rehr, J. Chem. Phys. 60, 1297 (1974).

${ }^{17}$ P. H. E. Meijer, R. Kikuchi, and P. Papon, Physica A 109, 365 (1981).

${ }^{18}$ H. E. Stanley and J. Teixeira, J. Chem. Phys. 73, 3404 (1980).

${ }^{19}$ H. A. Kramers and G. H. Wannier, Phys. Rev. 60, 252 (1941).

${ }^{20}$ R. Kikuchi, J. Chem. Phys. 60, 1071 (1974).

${ }^{21}$ R. Kikuchi and D. deFontaine, Scr. Met. 10, 995 (1976).

${ }^{22} \mathrm{R}$. Kikuchi and D. deFontaine in Applications of Phase Diagrams in Metallurgy and Ceramics, Natl. Bur. Stand. SP496 (1977), p. 967.

${ }^{23}$ G. Arfken, Mathematical Methods for Physicists (Academic, New York, 1970), p. 790

${ }^{24}$ R. van der Haegen, R. Luyckx, and H. N. W. Lekkerkerker, Physica A $107,622(1981)$.

${ }^{25}$ P. H. E. Meijer and W. C. Stamm, Physica A 90, 77 (1978)

${ }^{26}$ R. Kikuchi, J. Chem. Phys. 47, 1664 (1967).

${ }^{27}$ F. Broto, D. Clausse, L. Babin, and M. Clausse, Colloid Polym. Sci. 257, 302 (1979).

${ }^{28}$ R. D. Lang and R. D. Morgan, J. Chem. Phys. 73, 5849 (1980).

${ }^{29} \mathrm{E}$. Bodegom, Ph. D. thesis, Catholic University of America, University Microfilms, Ann Arbor, 1982.

${ }^{30}$ R. Kikuchi, J. Chem. Phys. 65, 4545 (1976).

${ }^{31}$ P. Henrici, Elements of Numerical Analysis (Wiley, New York, 1964). 